\title{
GLOBAL PINCHING THEOREMS OF SUBMANIFOLDS IN SPHERES
}

\author{
KAIREN CAI
}

Received 8 June 2001

Let $M$ be a compact embedded submanifold with parallel mean curvature vector and positive Ricci curvature in the unit sphere $S^{n+p}(n \geq 2, p \geq 1)$. By using the Sobolev inequalities of P. $\mathrm{Li}(1980)$ to $L_{p}$ estimate for the square length $\sigma$ of the second fundamental form and the norm of a tensor $\phi$, related to the second fundamental form, we set up some rigidity theorems. Denote by $\|\sigma\|_{p}$ the $L_{p}$ norm of $\sigma$ and $H$ the constant mean curvature of $M$. It is shown that there is a constant $C$ depending only on $n, H$, and $k$ where $(n-1) k$ is the lower bound of Ricci curvature such that if $\|\sigma\|_{n / 2}<C$, then $M$ is a totally umbilic hypersurface in the sphere $S^{n+1}$.

2000 Mathematics Subject Classification: 53C20, 53C40.

1. Introduction. Let $M$ be an $n$-dimensional compact oriented submanifold embedded in the unit sphere $S^{n+p}(1)$ of the Euclidean space $\mathbb{R}^{n+p+1}$. Inspiring the well-known results about minimal submanifolds due to Simons [7], some authors [5, 6, 8] have considered the estimate by using $L_{p}$ norm of the geometric objects instead of pointwise to study the geometric property of submanifolds. Shen [6] has obtained some global pinching theorems for minimal hypersurfaces in sphere. As a natural generalization of minimal submanifold, the submanifold with parallel mean curvature vector is one of the problems which received a great amount of attention recent years. Attempts have been made to extend Shen's result to hypersurfaces with constant mean curvature $H$, but so far we know nothing is reached. Also, we notice that Alencar and do Carmo [1] study hypersurfaces with constant mean curvature $H$ by using a significant method of introducing the tensor $\phi$, related to $H$ and to the second fundamental form. Along this direction, we will deal with the case of constant mean curvature and give some global pinching results. Furthermore, we successfully generalize these results to the case of submanifolds in sphere. By means of $L_{p}$ estimate for the norm of tensor $\phi$ and the square length $\sigma$ of the second fundamental form, we prove the following global pinching theorems.

THEOREM 1.1. Let $M$ be an $n$-dimensional compact submanifold embedded in the sphere $S^{n+p}(1)(n \geq 3, p \geq 1)$. Suppose that $M$ has parallel mean curvature vector and positive Ricci curvature. Denote by $H$ the mean curvature, $\sigma$ the square length of the second fundamental form, and $(n-1) k$ the lower bound of Ricci curvature of $M$. Then, there is a constant $C$ depending only on $n, H$, and $k$ (see (3.7) below) such that if $\left(\int_{M} \sigma^{n / 2}\right)^{2 / n}<C, M$ must be a totally umbilic hypersurface in the sphere $S^{n+1}(1)$.

THEOREM 1.2. Let $M$ be a compact surface with parallel mean curvature vector and zero genus embedded in sphere $S^{p+2}(1)(p \geq 1)$. Suppose that the Gauss curvature of 
$M$ has a positive lower bound $k$. If $\int_{M} \sigma^{2}<4 k^{7} / \pi^{11}(11+6 \sqrt{2})\left(1+H^{2}\right)^{6}$, where $H$ is the mean curvature and $\sigma$ is the square length of the second fundamental form of $M$, then $M$ is a totally umbilic surface in the sphere $S^{3}(1)$.

2. Preliminaries. The Sobolev inequality obtained by Li [4] states: suppose that $M$ is a compact connected Riemannian manifold. For every $f \in H_{1,2}\left(M^{n}\right), n=\operatorname{dim} M>2$, we have

$$
\int_{M}|\nabla f|^{2} \geq\left(\frac{n-2}{2(n-1)}\right)^{2} C_{0}^{2 / n}\left\{2^{-(n+2) / n}\|f\|_{2 n /(n-2)}^{2}-(\operatorname{vol} M)^{-2 / n} 2^{E(n)}\|f\|_{2}^{2}\right\},
$$

where

$$
\begin{aligned}
& \|f\|_{p}=\left(\int_{M}|f|^{p}\right)^{1 / p}, \\
& E(n)= \begin{cases}\frac{(n-4)(n-2)}{2}, & \text { if } n>3, \\
1, & \text { if } n=3\end{cases}
\end{aligned}
$$

and the best Sobolev constant $C_{0}$ satisfies

$$
C_{1} \leq C_{0} \leq 2 C_{1} .
$$

In (2.4)

$$
C_{1}=\inf \frac{(\operatorname{Area}(S))^{n}}{\left(\min \left(\operatorname{vol} M_{1}, \operatorname{vol} M_{2}\right)\right)^{n-1}}
$$

is the isoperimetric constant of $M^{n}$ where $S$ takes all hypersurfaces of $M, S$ divides $M$ into two parts $M_{1}$ and $M_{2}$, and $\operatorname{Area}(S)$ is the $(n-1)$-dimensional volume of $S$. Let

$$
k_{1}=2^{-3-2 / n}\left(\frac{n-2}{n-1}\right)^{2} C_{1}^{2 / n}, \quad k_{2}=2^{E(n)+2 / n-2}\left(\frac{n-2}{n-1}\right)^{2} C_{1}^{2 / n}(\operatorname{vol} M)^{-2 / n},
$$

then we have

$$
\int_{M}|\nabla f|^{2} \geq k_{1}\|f\|_{2 n /(n-2)}^{2}-k_{2}\|f\|_{2}^{2} .
$$

Let $S^{n+p}$ be an $(n+p)$-dimensional standard sphere in the Euclidean space $\mathbb{R}^{n+p+1}$ and $M$ a compact submanifold isometrically immersed in $S^{n+p}(1)$. We choose a local field of orthonormal frames $\left\{e_{A}\right\}, 1 \leq A \leq n+p$, in $S^{n+p}$ such that, restricted to $M$, the vectors $\left\{e_{i}\right\}, 1 \leq i \leq n$, are tangent to $M$. We denote the second fundamental form of $M$ by

$$
B=\sum_{i, j, \alpha} h_{i j}^{\alpha} \omega_{i} \otimes \omega_{j} \otimes e_{\alpha},
$$

where, $1 \leq i, j \leq n, n+1 \leq \alpha \leq n+p,\left\{\omega_{i}\right\}$ is the dual frame of $\left\{e_{i}\right\}$. The Weingarten transformation $H_{\alpha}$ corresponding to the normal vector $e_{\alpha}$ is defined by using the Riemannian metric of $M$

$$
\left\langle H_{\alpha}(X), Y\right\rangle=\left\langle B(X, Y), e_{\alpha}\right\rangle,
$$


where $X, Y$ are the tangent vectors of $M$. Denote the mean curvature vector of $M$ by

$$
\xi=\frac{1}{n} \sum_{\alpha}\left(\operatorname{tr} H_{\alpha}\right) e_{\alpha}
$$

where $\operatorname{tr} H_{\alpha}$ is the trace of the transformation $H_{\alpha}$. Then, the mean curvature $H$ and the square length $\sigma$ of the second fundamental form of $M$ can be expressed as

$$
H=|\xi|=\frac{1}{n} \sqrt{\sum_{\alpha}\left(\operatorname{tr} H_{\alpha}\right)^{2}}, \quad \sigma=\sum_{\alpha} \operatorname{tr}\left(H_{\alpha}\right)^{2} .
$$

If we choose $e_{n+1}$ such that $H e_{n+1}=\xi$, then

$$
\operatorname{tr} H_{n+1}=n H, \quad \operatorname{tr} H_{\beta}=0, \quad n+2 \leq \beta \leq n+p .
$$

Furthermore, $M$ is totally umbilical if and only if $M$ is umbilical with respect to any unit normal vector; $M$ is called a manifold with parallel mean curvature vector if it is parallel in normal bundle of $M$, that is, $\nabla_{X}^{\perp} \xi=0$ for any tangent vector $X$ of $M$ where $\nabla^{\perp}$ is the connection of the normal bundle of $M$. At this time, it is easy to check that the mean curvature of $M$ is constant from $\nabla_{X}(\xi, \xi)$. Let

$$
|\psi|^{2}=\sum_{\beta \neq n+1} \operatorname{tr}\left(H_{\beta}\right)^{2} .
$$

We know from [9, page 80] that if $M$ has parallel mean curvature normal vector, then the Laplacian of function $|\psi|^{2}$ satisfies the inequality

$$
\begin{aligned}
\frac{1}{2} \Delta|\psi|^{2} & \geq \sum_{i, j, k, \beta}\left(h_{i j k}^{\beta}\right)^{2}+n|\psi|^{2}-\left(3+\sqrt{n}-\frac{1}{p-1}\right) \sigma|\psi|^{2} \\
& \geq|\nabla| \psi||^{2}+n|\psi|^{2}-(3+\sqrt{n}) \sigma|\psi|^{2},
\end{aligned}
$$

where $1 \leq i, j, k \leq n, n+2 \leq \beta \leq n+p$.

3. Lemmas. First of all, we set up a lower bound depending only on $n, H$, and $k$ for $k_{1}$ and $k_{1} / k_{2}$ in the Sobolev inequality (2.7) of Li [4].

LEMMA 3.1. Let $M^{n}(n \geq 3)$ be a compact hypersurface with constant mean curvature $H$ embedded in the unit sphere $S^{n+1}(1)$. Suppose that the Ricci curvature of $M$ has a positive lower bound $(n-1) k$. Then, there is a positive constant $C$ depending only on $n, H$, and $k$ such that

$$
C<\min \left\{\frac{2 \sqrt{n-1}}{n} k_{1}, 2 \sqrt{n-1} \frac{k_{1}}{k_{2}}\right\},
$$

where $k_{1}$ and $k_{2}$ are defined in (2.6).

PRoof. Since $M$ has positive Ricci curvature, a result due to Croke [3] shows that the inequality

$$
C_{1}(M) \geq \frac{(\operatorname{vol} M)^{n+1}}{4 \omega_{n-1} \omega_{n}^{n-1}}\left(\frac{1}{\int_{0}^{d}(\sqrt{1 / k} \sin \sqrt{k} r)^{n-1} d r}\right)^{n+1} \geq \frac{n^{n+1}(\operatorname{vol} M)^{n+1}}{4 d^{n(n+1)} \omega_{n-1} \omega_{n}^{n-1}}
$$


holds for $n \geq 2$, where $d$ is the diameter of $M$ and $\omega_{n}$ is the volume of the unit sphere $S^{n}(1)$. It follows from Myers theorem that $d \leq \pi / \sqrt{k}$ for a compact manifold whose Ricci curvature has positive lower bound $(n-1) k$.

Then, we have

$$
C_{1}(M) \geq \frac{n^{n+1} k^{n(n+1) / 2}}{4 \pi^{n(n+1)}} \frac{(\operatorname{vol} M)^{n+1}}{\omega_{n-1} \omega_{n}^{n-1}} .
$$

From a result of Chen [2], we know that for any $p$-dimensional compact submanifold $\bar{M}$ in the Euclidean space $\mathbb{R}^{m}$, we have

$$
\int_{\bar{M}}|\bar{H}|^{p} \geq \omega_{p},
$$

where $\bar{H}$ is the mean curvature of $\bar{M}$ in $R^{m}$ and $\omega_{p}$ is the volume of the unit sphere $S^{p}(1)$. In our case that $M$ is embedded in the unit sphere $S^{n+1}(1)$, we have $\bar{H}^{2}=1+H^{2}$, where $H$ is the constant mean curvature of $M$ in $S^{n+1}(1)$. Therefore,

$$
\operatorname{vol} M \geq \frac{\omega_{n}}{\left(1+H^{2}\right)^{n / 2}} .
$$

It derives from (3.3) that

$$
C_{1}(M) \geq \frac{n^{n+1} \omega_{n}^{2}}{4 \omega_{n-1}}\left(\frac{k}{\pi^{2}\left(1+H^{2}\right)}\right)^{n(n+1) / 2} .
$$

Thus, $2 \sqrt{n-1} k_{1} / n$ has a positive lower bound depending only on $n, H$, and $k$, so does $2 \sqrt{n-1} k_{1} / k_{2}$. By a direct calculation from (2.6), (3.5), and (3.6), we can obtain the following constant which is only depending on $n, H$, and $k$ :

$$
\begin{aligned}
\min & \left\{2^{-2-6 / n} n^{1+2 / n} \frac{(n-2)^{2}}{(n-1)^{3 / 2}}\left(\frac{\omega_{n}^{2}}{\omega_{n-1}}\right)^{2 / n}\left(\frac{k}{\pi^{2}\left(1+H^{2}\right)}\right)^{n+1},\right. \\
& \left.2^{-E(n)-4 / n} \sqrt{n-1} \frac{\omega_{n}^{2 / n}}{1+H^{2}}\right\} \\
\leq & \min \left\{\frac{2 \sqrt{n-1}}{n} k_{1}, 2 \sqrt{n-1} \frac{k_{1}}{k_{2}}\right\} .
\end{aligned}
$$

Applying Lemma 3.1, we can prove the following lemma.

LEMMA 3.2. Let $M^{n}(n \geq 3)$ be a compact hypersurface embedded in the unit sphere $S^{n+1}(1)$. The hypersurface $M$ possesses constant mean curvature $H$ and positive Ricci curvature. Denote by $\sigma$ the square length of the second fundamental form and by $(n-1) k$ the lower bound of Ricci curvature of $M$. Then, there is a constant $C$ depending only on $n, H$, and $k$ (see (3.7) above) such that if $\left(\int_{M} \sigma^{n / 2}\right)^{2 / n}<C, M$ must be a totally umbilic hypersurface in $S^{n+1}(1)$.

Proof. We define a linear map $\phi: T_{p} M \rightarrow T_{p} M$ by

$$
\langle\phi X, Y\rangle=H\langle X, Y\rangle-\langle A X, Y\rangle,
$$


where $A$ is the Weingarten transformation for the normal vector of the hypersurface. It is easily checked that $\operatorname{tr} \phi=0$ and $|\phi|^{2}=\sigma-n H^{2}$ where $\operatorname{tr} \phi$ is the trace of $\phi$ and $\sigma$ is the square length of the second fundamental form of $M$. Furthermore, $|\phi|^{2}=0$ if and only if $M$ is totally umbilic. Since $M$ possesses constant mean curvature, we have the following identity (see [1, page 1225])

$$
\frac{1}{2} \Delta|\phi|^{2}=|\nabla \phi|^{2}-|\phi|^{4}+n\left(1+H^{2}\right)|\phi|^{2}-n H \operatorname{tr} \phi^{3} .
$$

We have obtained the following estimate in ([1, page 1226]).

$$
\frac{1}{2} \Delta|\phi|^{2} \geq|\nabla \phi|^{2}+|\phi|^{2}\left(n\left(1+H^{2}\right)-\frac{n-2}{\sqrt{n-1}} \sqrt{n H^{2}}|\phi|-|\phi|^{2}\right) .
$$

Consider a quadratic form with the eigenvalues $\pm n / 2 \sqrt{n-1}$

$$
F\left(\sqrt{n H^{2}},|\phi|\right)=n H^{2}-\frac{n-2}{\sqrt{n-1}} \sqrt{n H^{2}}|\phi|-|\phi|^{2} .
$$

Then, there is an orthogonal transformation $\psi:\left(\sqrt{n H^{2}},|\phi|\right) \rightarrow(u, v)$ such that

$$
F\left(\sqrt{n H^{2}},|\phi|\right)=\frac{n}{2 \sqrt{n-1}}\left(u^{2}-v^{2}\right) .
$$

It follows from $u^{2}+v^{2}=n H^{2}+|\phi|^{2}=\sigma$ that

$$
F\left(\sqrt{n H^{2}},|\phi|\right) \geq-\frac{n}{2 \sqrt{n-1}}\left(u^{2}+v^{2}\right)=-\frac{n}{2 \sqrt{n-1}} \sigma .
$$

Because $|\nabla| \phi||^{2} \leq|\nabla \phi|^{2}$, so

$$
\frac{1}{2} \Delta|\phi|^{2} \geq|\nabla| \phi||^{2}+n|\phi|^{2}-\frac{n}{2 \sqrt{n-1}} \sigma|\phi|^{2} .
$$

It follows from (2.7) that

$$
\int_{M}|\nabla| \phi\left\|^{2} \geq k_{1}\right\| \phi\left\|_{2 n /(n-2)}^{2}-k_{2}\right\| \phi \|_{2}^{2},
$$

where $k_{1}, k_{2}$ have been defined in (2.6). Integrating both sides of (3.14) and applying (3.15) and the inequality

$$
\left\|\sigma|\phi|^{2}\right\|_{1} \leq\|\sigma\|_{n / 2}\|\phi\|_{2 n /(n-2)}^{2},
$$

we get

$$
\begin{aligned}
0 & \geq\left(n-k_{2}\right)\|\phi\|_{2}^{2}+k_{1}\|\phi\|_{2 n /(n-2)}^{2}-\frac{n}{2 \sqrt{n-1}}\left\|\sigma|\phi|^{2}\right\|_{1} \\
& \geq\left(n-k_{2}\right)\|\phi\|_{2}^{2}+\left(k_{1}-\frac{n}{2 \sqrt{n-1}}\|\sigma\|_{n / 2}\right)\|\phi\|_{2 n /(n-2)}^{2} .
\end{aligned}
$$

Suppose that

$$
\|\sigma\|_{n / 2}<\min \left\{\frac{2 \sqrt{n-1}}{n} k_{1}, 2 \sqrt{n-1} \frac{k_{1}}{k_{2}}\right\} .
$$


By integrating both sides of (3.14) and applying (3.18), we obtain that

$$
0 \geq n\|\phi\|_{2}^{2}-\frac{n}{2 \sqrt{n-1}}\|\phi\|_{2 n /(n-2)}^{2}\|\sigma\|_{n / 2} \geq n\|\phi\|_{2}^{2}-n \frac{k_{1}}{k_{2}}\|\phi\|_{2 n /(n-2)}^{2} .
$$

If $|\phi|^{2} \neq 0$, it follows from (3.17), (3.18), and (3.19) that

$$
\begin{aligned}
0 & \geq\left(n-k_{2}\right)\|\phi\|_{2}^{2}+\left(k_{1}-\frac{n}{2 \sqrt{n-1}}\|\sigma\|_{n / 2}\right) \frac{k_{2}}{k_{1}}\|\phi\|_{2}^{2} \\
& \geq\left(n-\frac{n}{2 \sqrt{n-1}} \frac{k_{2}}{k_{1}}\|\sigma\|_{n / 2}\right)\|\phi\|_{2}^{2}>0 .
\end{aligned}
$$

A contradiction appears. Hence $|\phi|^{2}=0$, that is, $M$ is totally umbilic. From Lemma 3.1, we conclude that Lemma 3.2 holds.

In the case of $n=2$, we have the following lemma.

LEMMA 3.3. Let $M$ be a compact surface with constant mean curvature $H$ and zero genus embedded in the sphere $S^{3}(1)$. Suppose that the Gauss curvature of $M$ has a positive lower bound $k$. If $\int_{M}\left(\sigma-2 H^{2}\right)^{2}<4 k^{7} / \pi^{11}\left(1+H^{2}\right)^{6}$, then $\sigma-2 H^{2}=0$, that is, $M$ is a totally umbilic surface.

Proof. When $\operatorname{dim} M=2$, it immediately follows from (3.9) that

$$
\frac{1}{2} \Delta|\phi|^{2}=|\nabla \phi|^{2}+2\left(1+H^{2}\right)|\phi|^{2}-|\phi|^{4} .
$$

Furthermore, Li [4] obtained another Sobolev inequality for $\operatorname{dim} M=2$ : for every $f \in$ $H_{1,2}\left(M^{2}\right)$, we have

$$
\begin{aligned}
\int_{M}|\nabla f|^{2} & \geq \frac{C_{0}}{4}\left\{(\operatorname{vol} M)^{-1 / 2}\left(\int_{M} f^{4}\right)^{1 / 2}-(\operatorname{vol} M)^{-1} \int_{M} f^{2}\right\} \\
& \geq \tilde{k}_{1}\|f\|_{4}^{2}-\tilde{k}_{2}\|f\|_{2}^{2},
\end{aligned}
$$

where

$$
\tilde{k}_{1}=\frac{C_{1}}{4}(\operatorname{vol} M)^{-1 / 2}, \quad \tilde{k}_{2}=\frac{C_{1}}{2}(\operatorname{vol} M)^{-1},
$$

and $C_{0}$ is the best Sobolev constant, $C_{1}$ the isoperimetric constant of $M, C_{1} \leq C_{0} \leq 2 C_{1}$. Because $|\nabla| \phi||^{2} \leq|\nabla \phi|^{2}$, integrating both sides of (3.21) and applying (3.22), we get

$$
0 \geq\left(2+2 H^{2}-\tilde{k}_{2}\right)\|\phi\|_{2}^{2}+\left(\tilde{k}_{1}-\|\phi\|_{4}^{2}\right)\|\phi\|_{4}^{2} .
$$

Suppose that

$$
\|\phi\|_{4}^{2}<\min \left\{\tilde{k}_{1}, 2\left(1+H^{2}\right) \frac{\tilde{k}_{1}}{\tilde{k}_{2}}\right\} .
$$

It follows from (3.21) that

$$
0 \geq 2\left(1+H^{2}\right)\|\phi\|_{2}^{2}-\|\phi\|_{4}^{4} .
$$


Hence,

$$
\frac{\tilde{k}_{2}}{\tilde{k}_{1}}\|\phi\|_{2}^{2} \leq\|\phi\|_{4}^{2} .
$$

If $|\phi|^{2} \neq 0$ from (3.24), (3.25), and (3.27), we derive

$$
0 \geq\left(2+2 H^{2}-\frac{\tilde{k}_{2}}{\tilde{k}_{1}}\|\phi\|_{4}^{2}\right)\|\phi\|_{2}^{2}>0 .
$$

This is a contradiction.

We estimate the lower bound of the isoperimetric constant $C_{1}$ and the upper bound of the volume of $M$ to obtain a lower bound of $\min \left\{\tilde{k}_{1},\left(2+2 H^{2}\right) \tilde{k}_{1} / \tilde{k}_{2}\right\}$ which is only depending on $H$ and $k$. We will make use of the same argument as Wang's in [8]. In fact, from (3.5) it is true that

$$
\operatorname{vol} M \geq \frac{\omega_{2}}{1+H^{2}}=\frac{4 \pi}{1+H^{2}}
$$

It follows from (3.6) that

$$
C_{1}(M) \geq \frac{16 k^{3}}{\pi^{5}\left(1+H^{2}\right)^{3}}
$$

According to Gauss-Bonnet formula, we have

$$
k \operatorname{vol} M \leq \int_{M} K d V=2 \pi \chi(M)=4 \pi,
$$

where $\chi(M)$ is the Euler character of surface $M$. Thus, both $\tilde{k}_{1}$ and $\tilde{k}_{1} / \tilde{k}_{2}$ have positive lower bounds depending only on $H$ and $k$. By a direct calculation from (3.23), (3.29), (3.30), and (3.31), we get

$$
\frac{2 k^{7 / 2}}{\pi^{11 / 2}\left(1+H^{2}\right)^{3}} \leq \min \left\{\tilde{k}_{1}, 2\left(1+H^{2}\right) \frac{\tilde{k}_{1}}{\tilde{k}_{2}}\right\} .
$$

This completes the proof of Lemma 3.3.

\section{Proof of theorems}

Proof OF TheOrem 1.1. Lemmas 3.2 and 3.3 imply that Theorems 1.1 and 1.2 hold when the codimension $p=1$. Now, we need to consider the case of $p>1$. By using the same argument as in estimating $|\phi|^{2}$ satisfying (3.14) and (3.15) to $|\psi|^{2}$ in (2.14) with $n>2$, we obtain that if

$$
\|\sigma\|_{n / 2}<\min \left\{\frac{k_{1}}{(3+\sqrt{n})}, \frac{n k_{1}}{(3+\sqrt{n}) k_{2}}\right\},
$$


then $|\psi|^{2}=0$. It is easy to see from a theorem of Erbacher (see [9, page 80]) that $M$ lies in a totally geodesic sphere $S^{n+1}(1)$. From (3.7), it is easy to check that

$$
\begin{aligned}
& \min \left\{2^{-3-6 / n} \frac{n^{2+2 / n}}{3+\sqrt{n}}\left(\frac{n-2}{n-1}\right)^{2}\left(\frac{\omega_{n}^{2}}{\omega_{n-1}}\right)^{2 / n}\left(\frac{k}{\pi^{2}\left(1+H^{2}\right)}\right)^{n+1},\right. \\
& \left.2^{-E(n)-1-4 / n} \frac{n \omega_{n}^{2 / n}}{(3+\sqrt{n})\left(1+H^{2}\right)}\right\} \\
& \leq \min \left\{\frac{k_{1}}{3+\sqrt{n}}, \frac{n k_{1}}{(3+\sqrt{n}) k_{2}}\right\} \\
& \leq \min \left\{\frac{2 \sqrt{n-1}}{n} k_{1}, 2 \sqrt{n-1} \frac{k_{1}}{k_{2}}\right\},
\end{aligned}
$$

where $\omega_{n}$ is the volume of the unit sphere $S^{n}(1)$ and $E(n)$ is as shown in (2.3). As Lemma 3.2 has shown, we can conclude that if $\|\sigma\|_{n / 2}$ is less than the above constant depending only on $n, H$, and $k$, then $|\phi|^{2}=0$, that is, $M$ is a totally umbilic hypersurface in the sphere $S^{n+1}(1)$. This ends the proof.

Proof OF THeOrem 1.2. The argument is similar to the proof of Lemma 3.3. We only need to verify the case of $n=2$ and $p>1$ in (2.14). We get that

$$
\frac{1}{2} \Delta|\psi|^{2} \geq|\nabla \psi|^{2}+2|\psi|^{2}-(3+\sqrt{2}) \sigma|\psi|^{2} .
$$

Comparing with (3.21) and (3.25), we can get directly that, if

$$
\|\sigma\|_{2} \leq \min \left\{\frac{\tilde{k}_{1}}{3+\sqrt{2}}, \frac{2 \tilde{k}_{1}}{(3+\sqrt{2}) \tilde{k}_{2}}\right\},
$$

then $|\psi|^{2}=0, M$ lies in $S^{3}(1)$. So from (3.32), we have

$$
\frac{2 k^{7 / 2}}{\pi^{11 / 2}(3+\sqrt{2})\left(1+H^{2}\right)^{3}} \leq \min \left\{\frac{\tilde{k}_{1}}{3+\sqrt{2}}, \frac{2\left(1+H^{2}\right) \tilde{k}_{1}}{(3+\sqrt{2}) \tilde{k}_{2}}\right\} .
$$

Furthermore, if $\int_{M} \sigma^{2}<4 k^{7} / \pi^{11}(11+6 \sqrt{2})\left(1+H^{2}\right)^{6}$, it follows that

$$
\int_{M}\left(\sigma-2 H^{2}\right)^{2} \leq \int_{M} \sigma^{2} \leq \frac{4 k^{7}}{\pi^{11}(11+6 \sqrt{2})\left(1+H^{2}\right)^{6}} \leq \frac{4 k^{7}}{\pi^{11}\left(1+H^{2}\right)^{6}} .
$$

From Lemma 3.3, we obtain that $M$ is a totally umbilic surface in $S^{3}(1)$.

REMARK 4.1. We should point out that we do not know whether [8, Theorem 3] is correct, there is a wrong calculation in the proof of [8, Lemma 2] (see [8, formula (3)]). This mistake also influences all constants $A(n, p, c)$ used in all theorems in [8], but it does not affect the generalization of Theorems 1.1 and 1.2 in this paper. In fact, a slight modification for [8, Lemma 2] is needed and the correct one is (4.1) of this paper.

REMARK 4.2. In the case of a minimal submanifold of a sphere, that is, $H=0$, we will have no problem to draw and prove similar conclusions as Theorems 1.1 and 1.2. 
Since a minimal and totally umbilic hypersurface is totally geodesic, so these rigidity theorems can be treated as a direct generalization of Shen's results which dealt with the case of codimension $p=1$ and mean curvature $H=0$ [6].

AcknOwLEDGments. The author is grateful to the School of Mathematics, University of Bristol for their hospitality during his visit. This work is supported by the National Natural Science Foundation of China.

\section{REFERENCES}

[1] H. Alencar and M. do Carmo, Hypersurfaces with constant mean curvature in spheres, Proc. Amer. Math. Soc. 120 (1994), no. 4, 1223-1229.

[2] B.-Y. Chen, Total Mean Curvature and Submanifolds of Finite Type, Series in Pure Mathematics, vol. 1, World Scientific Publishing, Singapore, 1984.

[3] C. B. Croke, Some isoperimetric inequalities and eigenvalue estimates, Ann. Sci. École Norm. Sup. (4) 13 (1980), no. 4, 419-435.

[4] P. Li, On the Sobolev constant and the p-spectrum of a compact Riemannian manifold, Ann. Sci. École Norm. Sup. (4) 13 (1980), no. 4, 451-468.

[5] J. M. Lin and C. Yu. Xia, Global pinching theorems for even-dimensional minimal submanifolds in the unit spheres, Math. Z. 201 (1989), no. 3, 381-389.

[6] C. L. Shen, A global pinching theorem of minimal hypersurfaces in the sphere, Proc. Amer. Math. Soc. 105 (1989), no. 1, 192-198.

[7] J. Simons, Minimal varieties in Riemannian manifolds, Ann. of Math. (2) 88 (1968), 62-105.

[8] H. Wang, Some global pinching theorems for submanifolds of a sphere, Acta Math. Sinica 31 (1988), no. 4, 503-509 (Chinese).

[9] S. T. Yau, Submanifolds with constant mean curvature. II, Amer. J. Math. 97 (1975), 76-100.

Kairen Cai: Department of Mathematics, Hangzhou Teacher's College, 96 Wen Yi ROAD, HANGZHOU 310036, CHINA

E-mail address: kcai@mai1.hz.zj.cn 


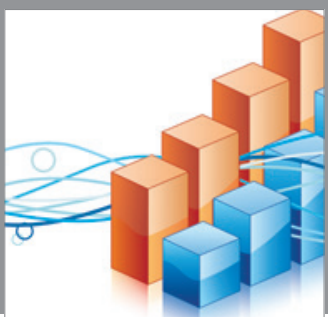

Advances in

Operations Research

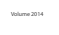

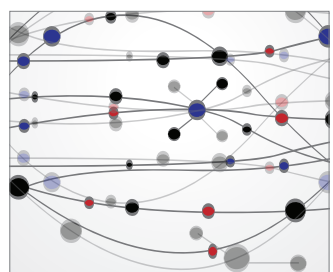

\section{The Scientific} World Journal
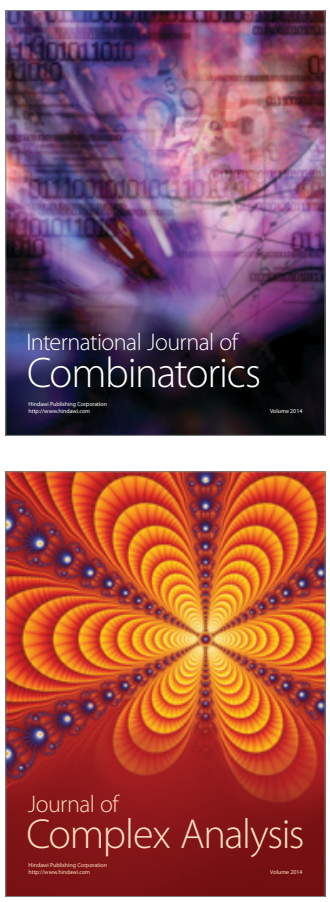

International Journal of

Mathematics and

Mathematical

Sciences
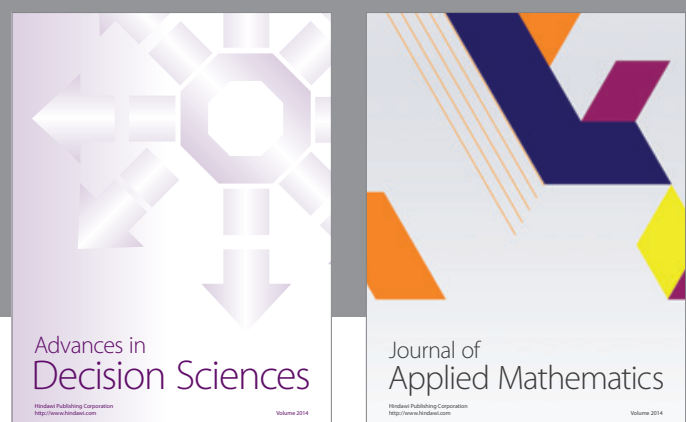

Journal of

Applied Mathematics
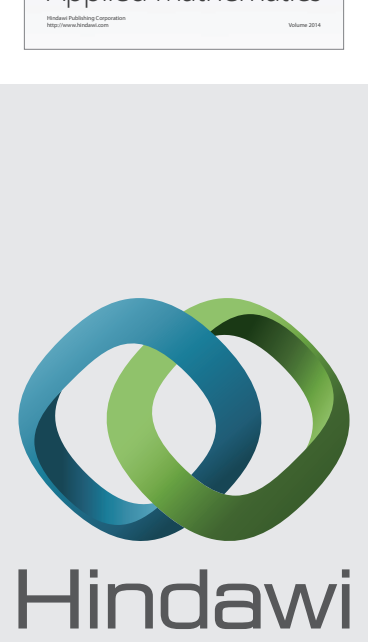

Submit your manuscripts at http://www.hindawi.com
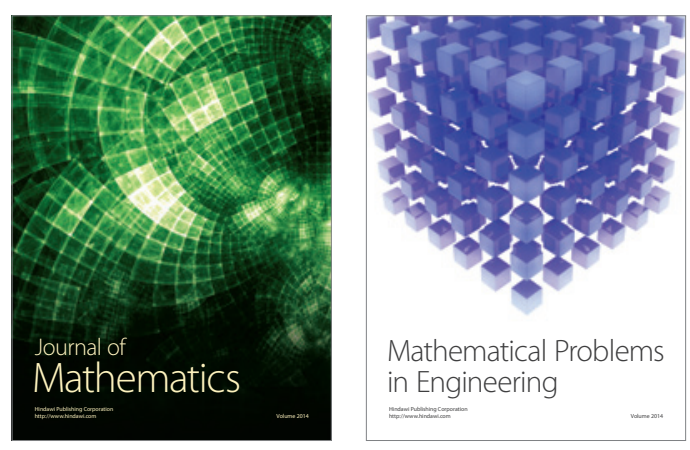

Mathematical Problems in Engineering
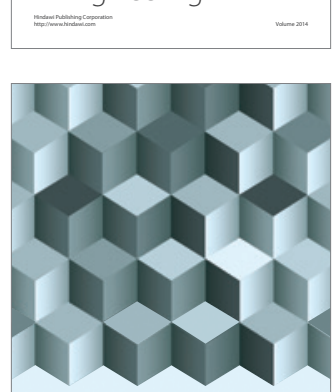

Journal of

Function Spaces
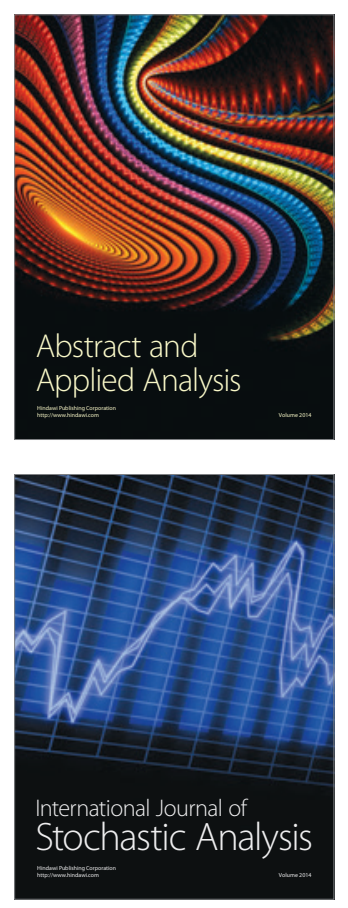

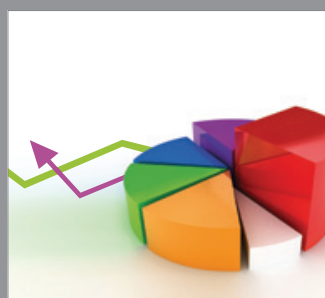

ournal of

Probability and Statistics

Promensencen
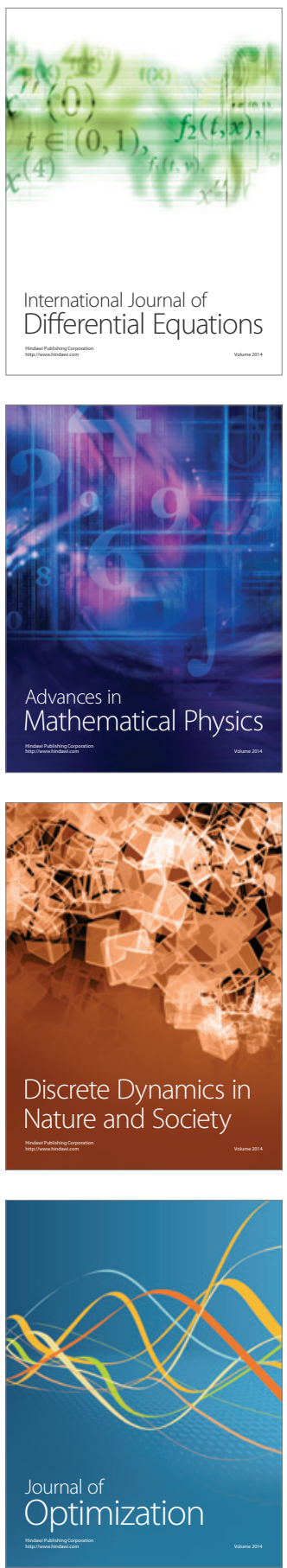